\title{
Uniparental disomy explains the occurrence of the Angelman or Prader-Willi syndrome in patients with an additional small inv $\operatorname{dup}(15)$ chromosome
}

Wendy P Robinson, Joseph Wagstaff, Fabiana Bernasconi, Carlo Baccichetti, Lina Artifoni, Emilio Franzoni, Lorraine Suslak, Ling-Yu Shih, Hannah Aviv, Albert A Schinzel

\begin{abstract}
A patient with Angelman syndrome and a 46,XY/47,XY, + inv dup(15)(pter $\rightarrow$ q11: q11 $\rightarrow$ pter) karyotype and a patient with Prader-Willi syndrome and a $46, X Y / 47, X Y,+$ inv $\operatorname{dup}(15)($ pter $\rightarrow$ q12: q12 $\rightarrow$ pter) karyotype were investigated with molecular markers along chromosome 15. Paternal uniparental isodisomy was found for all informative markers in the first case which indicates that this, rather than the presence of the extra chromosome, is the cause of the Angelman syndrome phenotype. Similarly, the PWS patient showed maternal uniparental disomy with absence of PWS region material on the inv dup(15) chromosome. If (1) marker chromosomes are an occasional by product of 'rescuing' a trisomic fertilisation, or (2) if duplication of the normal homologue in a zygote which has inherited a marker in place of the normal corresponding chromosome 'rescues' an aneuploid fertilisation, or (3) if the presence or formation of a marker chromosome increases the probability of non-disjunction, then uniparental disomy might be found occasionally in other subjects with de novo marker chromosomes.

(f Med Genet 1993;30:756-60)
\end{abstract}

Angelman syndrome is associated with a maternal deletion of $15 \mathrm{q} 11-\mathrm{q} 13$ in more than $50 \%$ of cases $^{1}$ or, much more rarely, paternal uniparental disomy (UPD) of chromosome 15 is found. ${ }^{2-4}$ Prader-Willi syndrome patients show a paternally derived deletion of the same region in about $75 \%$ of cases with maternal $\mathrm{UPD}(15)$ in virtually all remaining typical patients. ${ }^{5-7}$ This pattern of inheritance implies that there are at least two imprinted genes in this region, one active only on the maternally derived chromosome 15 and another active only on the paternally derived one.

The presence of a small extra marker chromosome derived from chromosome 15 is an occasional finding in Prader-Willi syndrome patients, ${ }^{8-14}$ but has not previously been reported in Angelman syndrome. These marker chromosomes are generally small and include only short arm material and occasionally some heterochromatic material from the $\mathrm{q}$ $\operatorname{arm}^{15}$ and are therefore unlikely to include the Prader-Willi syndrome critical region in
$15 \mathrm{q} 12$, as defined by minimum regions of deletion overlap in PWS patients.

It has been hypothesised previously by us that the karyotype of such patients probably originated as a trisomy for chromosome 15 with loss of one chromosome through marker formation occurring by an early postzygotic breakage/duplication event. ${ }^{16}$ If the marker formed from the single paternal chromosome, in a maternally derived trisomy 15 , this would result in maternal UPD(15) for the normal pair. This would be somewhat similar to the proposed mechanism to explain the observation that fetuses showing trisomy 15 in placental biopsy and a normal diploid karyotype at birth may occasionally show maternal UPD (15) and the associated PWS phenotype. ${ }^{1718}$ This hypothesis would predict that if the same mechanism occurred in a paternally derived trisomy 15 it would be possible to lose the single maternal chromosome, occasionally with gain of a maternally derived marker, resulting in paternal UPD for the normal pair of chromosomes 15 and the Angelman syndrome phenotype. An alternative hypothesis, also resulting in UPD(15), is that the presence or formation of a marker chromosome during meiosis may increase the probability of nondisjunction and therefore UPD. In this case the marker could originate from either parent since there is either a loss or gain of a chromosome 15 from both parents.

To investigate this possibility, we examined the inheritance of markers on the normal chromosomes 15 in an AS and a PWS patient both with a $46, \mathrm{XY} / 47, \mathrm{XY},+\operatorname{inv} \operatorname{dup}(15)$ karyotype.

\section{Methods and results}

CASE 1

The proband, a 4 year old male, was the only child born, after an uneventful pregnancy, to a 30 year old mother and 40 year old father. Birth weight was $2990 \mathrm{~g}$, birth length was $50 \mathrm{~cm}$, and head circumference was $34 \mathrm{~cm}$. At 6 months, a slow down in psychomotor acquisition was noted. He sat at 9 months and walked at 24 months. An abnormal EEG was found at 2 years and antiseizure medication was prescribed. A CT scan showed asymmetry of the lateral ventricles with the right ventricle more dilated than the left. Gait was unsteady, language was delayed, and trunk ataxia and tremors during fine movements were noted. 


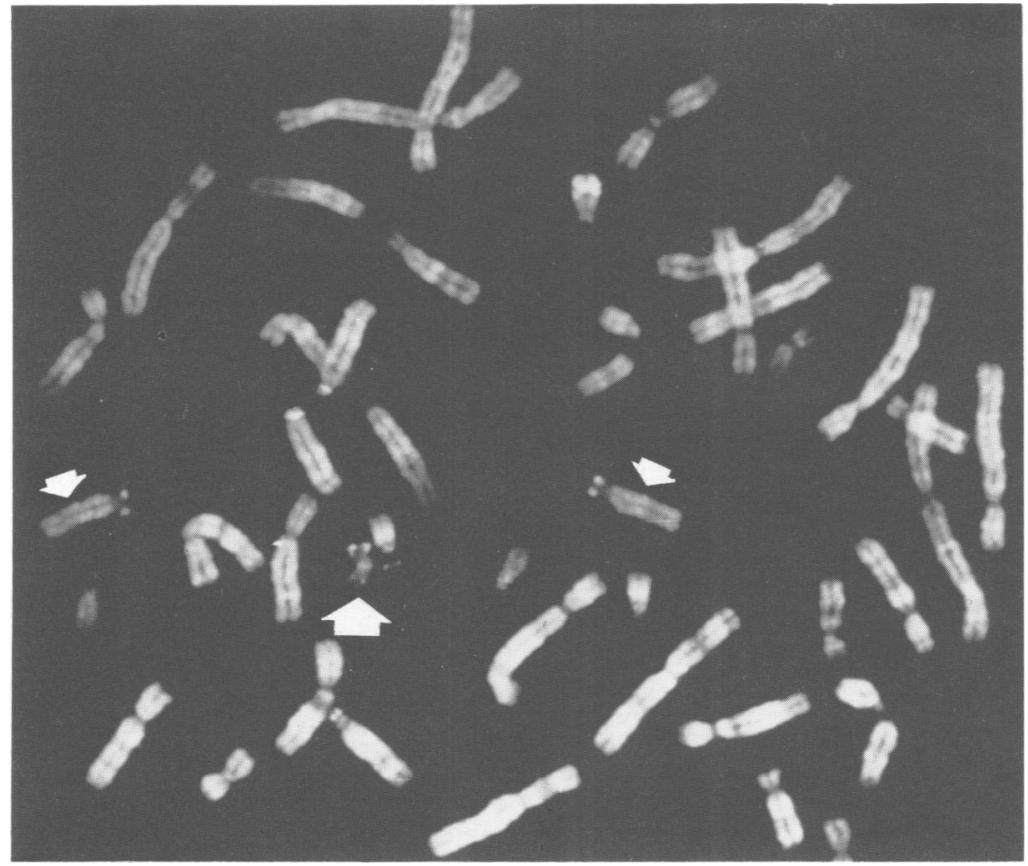

Figure $1 Q F Q$ stained metaphase from case 1. The supernumerary chromosome is indicated by larger arrow, smaller arrows point to the two normal chromosomes 15.

The external genitalia were normal. At 4 years the proband's height, weight, and head circumference were within the normal range. A clinical diagnosis of Angelman syndrome was made although no hypopigmentation was present.

\section{CASE 2}

At 12 years, this male patient showed all the classical signs of PWS including marked obesity resulting from hyperphagia, cryptorchidism, hypogenitalism, narrow forehead, and mental retardation. Neonatal hypotonia and feeding problems in infancy were present. Height was between the 10 th and 25 th centiles.

\section{CYTOGENETICS}

Cytogenetic analysis of a blood culture from case 1 showed the karyotype 46,XY/ $47, X Y,+\operatorname{inv} \operatorname{dup}(15)($ pter $\rightarrow$ q11:q11 $\rightarrow$ pter $)$ in a ratio of 40:60. Dystamycin-A DAPI staining showed two bright DAPI positive ends, indicating origin from chromosome 15. CBG staining showed that there were two distinct centromeres. Differential staining properties of the two ends of the marker were also seen with QFQ staining and indicate its origin from two different chromosomes 15 (fig 1). The inv $\operatorname{dup}(15)$ appears to contain very little of the $q$

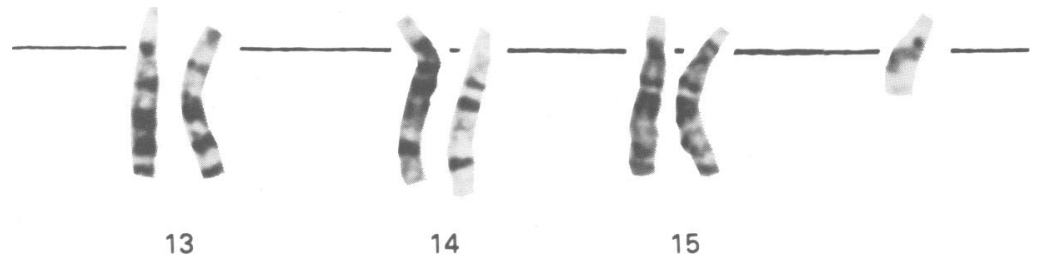

Figure 2 Partial $G$ banded karyotype from case 2 showing the D group chromosomes and marker. arm, and is presumably composed entirely of heterochromatic material. The chromosomes of the parents were normal.

Cytogenetic analysis of a blood culture from case 2 showed the karyotype 46,XY/ $47, X Y,+\operatorname{inv} \operatorname{dup}(15)$ in a ratio of 30:70. The inv dup(15) chromosome in this case is slightly larger and could be interpreted as inv $\operatorname{dup}(15)($ pter $\rightarrow$ q12:q12 $\rightarrow$ pter $)$ or inv $\operatorname{dup}(15)$ (pter $\rightarrow$ q11:q12 $\rightarrow$ pter) (fig 2). This chromosome, however, appears smaller than those we have analysed previously which contained one or two copies of the PWS region. ${ }^{16}$ The parental chromosomes were normal.

Material was no longer available in either case to perform in situ hybridisation of PWS/ AS region probes and thereby to confirm directly the content of the inv dup(15) chromosomes.

MOLECULAR ANALYSIS

Hybridisation of probes to DNA from the probands and parents were performed as described previously. ${ }^{6}$ Probes used included pIR39 (D15S18), p34 (D15S9), p3-21 (D15S10), pIR4-3 (D15S11), pIR10-1 (D15S12), and p189-1 (D15S13), ${ }^{19-21}$ all mapping within the PWS deletion region of 15q11-q13. In addition the VNTR probes CMW-1 (D15S24), ${ }^{22}$ located in 15q13, and ms620 (D15S86, mapping near the 15q telomere $)^{23}$ were also used. Microsatellite loci for D15S11, ${ }^{24}$ GABRB3, ${ }^{25}$ GABRA5, ${ }^{26}$ D15S97, CYP19, D15S108, ACTC, D15S87, and IPM15M9 (Genome data base) were detected by PCR amplification using ${ }^{32} \mathrm{P}$ labelled cytosine and visualised on a $6 \%$ polyacrylamide denaturing gel.

Case 1 showed no maternal inheritance for the D15S13 RFLP and the GABRB3 microsatellite loci, both mapping within the PWS/AS deletion region (fig 3A), and for the IPM15M9 microsatellite and D15S86 VNTR locus (fig 3B), mapping to distal chromosome 15 (table). Reduction of paternal heterozygosity to homozygosity in patient 1 was observed for all informative loci on chromosome 15: D15S18, GABRB3, GABRB5, D15S24, IPM15M9, and D15S86.

The father was not available for study in case 2 . However, the patient was identical to the mother at all loci, whereas the brother showed inheritance of non-maternal (paternal) alleles (table). Dosage analysis was performed with a Molecular Dynamics 'Personal Densitometer' and showed copy numbers in the patient of: IR39: 3.3; p34: 2.3; 28ß3-H3: 2.1,1.9 (two different blots); p189-1: 1.9; and 3-21:2.1 (fig 4). Thus, there was no evidence for a duplication of any loci except for the most proximal locus detected by IR39 (D15S18), which is also often not included in the deletion in many PWS and AS deletion cases. ${ }^{12728}$ Because only $70 \%$ of cells contained the extra marker chromosome, an average dosage of 3.3 copies for $\mathrm{p} 39$ indicates that two copies of this locus were present on the inv dup(15) chromosome.

Methylation studies using hybridisation of 
A

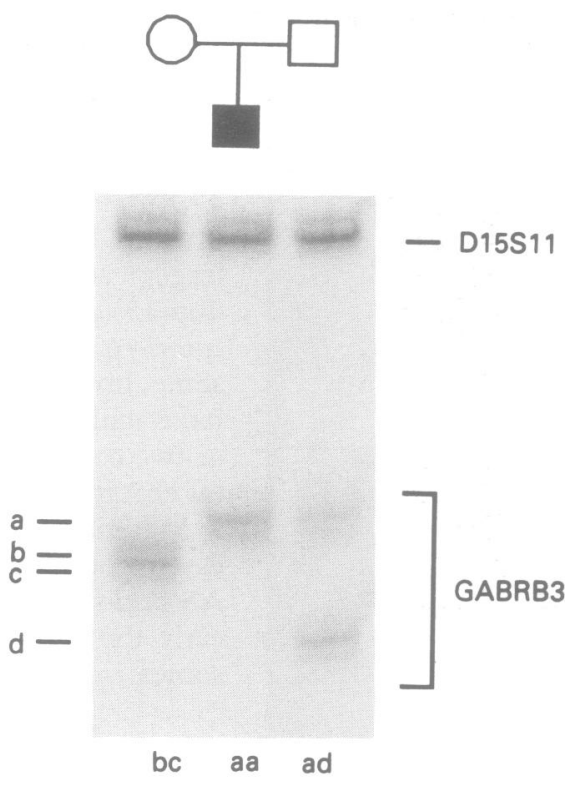

B

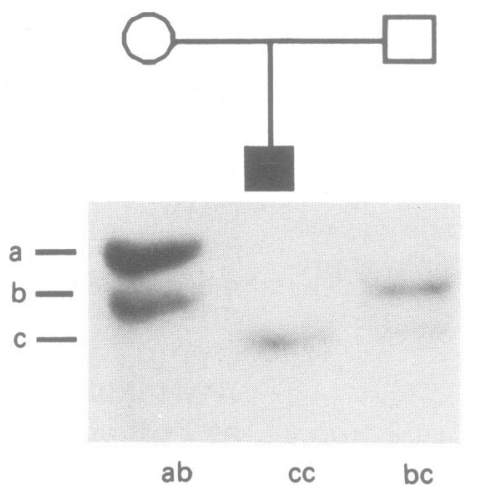
Figure 3 Molecular results supporting paternal
isodisomy of chromosome 15 in case 1. (A) PCR amplification was uninformative for the D15S11 microsatellite, but shows inheritance of a single paternal allele and no maternal allele at GABRB3. (B) Hybridisation of probe ms-620, mapping on distal $15 q$, shows only paternal inheritance.

PW71 to HindIII/HpaII digests were used to confirm the parental origin of the PWS region material. ${ }^{29}$ PW71 (locus D15S63) detects a parent of origin specific methylation pattern which has proven reliable in the diagnosis of deletions and UPD (15) involved in PWS and AS syndrome without requiring parental DNA. Our own unpublished results also indicate that the parent of origin methylation pattern is conserved in all rearrangements involving 15q11-13, including in eight patients with a maternally derived inv dup(15) chromosome that includes the PWS/AS region, in two patients with direct duplications of the PWS/AS region, in two cases with atypical PWS deletions, and in one ring(15) chromosome case.

In case 1 , only the unmethylated, paternal band was present, confirming that there was no maternal inheritance of PWS region genes. In case 2 , only the methylated maternal band was present, indicating that there was no paternal inheritance of PWS region genes (fig 5). As this patient is heterozygous in this PWS region and shows a normal diploid copy number, maternal uniparental heterodisomy of the two normal chromosome 15 homologues is the most likely explanation for the molecular results.

\section{Discussion}

Paternal isodisomy for the entire long arm of chromosome 15 was found in case 1 and is the explanation for the expression of the AS phenotype. The extra chromosome appears to contain mostly or only heterochromatic $p$ arm material, which should have no phenotypic effect. The parental origin of the marker chromosome could not be proven, although it seems likely to be maternal, since that is the chromosome 15 which is lacking. Maternal UPD(15) was found in the PWS patient, case 2. Although the father was not available for study, this diagnosis can be confirmed based on the observation of no paternal transmission determined using parent of origin methylation differences detectable by probe PW71, no evidence of a duplication (or a deletion) by dosage of probes in the PWS/AS critical region, and markers consistent with maternal UPD(15) throughout the chromosome 15.

The presence of homozygosity for markers along chromosome 15 in case 1 indicates that the second paternal chromosome 15 may have arisen as a postzygotic non-disjunction event rather than a meiotic one. In other words, the original karyotype in the zygote is likely to have been $46, X Y,-15,+\operatorname{inv} \operatorname{dup}(15)$, with rescue of the zygote by duplication of a single paternal chromosome. Instability of the marker could explain the mosaicism. Alternatively, the zygote may have originated as a trisomy with the maternal chromosome being lost through a breakage/duplication event. In this case, the paternal non-disjunction event must have been the result of either a meiotic I non-disjunction with recombination occurring only between D15S18 and the centromere or a meiotic II non-disjunction without meiosis I recombination. Lastly, the duplication of the father's chromosome and the formation of the inv dup(15) chromosome may have been related events.

The results of case 2 are consistent with origin from a trisomic conception with loss of the paternal chromosome 15 through a breakage/duplication event resulting in an inv $\operatorname{dup}(15)$ chromosome. ${ }^{16}$ It is also possible that the inv dup(15) chromosome was present before fertilisation in the gamete from either parent. Parental origin of the extra chromosome could not be determined. However, the second maternal chromosome presumably arose by a meiosis I non-disjunction event, as markers nearest to the centromere showed inheritance of both maternal alleles.

Although the majority of patients with small chromosomes derived from 15 which contain only heterochromatic material should be normal, such cases appear to be at an increased risk for maternal or paternal UPD(15) which would result in the PWS or AS phenotype. Thus, when such a finding occurs at prenatal 
Results of DNA analysis. Probes are shown in map order from centromere to telomere. Probes D15S9 to D15S12 are deleted in most PWS and AS deletion patients. Allele designations are arbitrary and are different for cases 1 and 2.

\begin{tabular}{|c|c|c|c|c|c|c|c|c|}
\hline \multirow[b]{2}{*}{ Locus } & \multirow[b]{2}{*}{ Probe/enzyme } & \multirow[b]{2}{*}{ Location } & \multicolumn{3}{|c|}{ Case 1} & \multicolumn{3}{|c|}{ Case 2} \\
\hline & & & Proband & Mother & Father & Proband & Mother & Brother \\
\hline D15S18 & 39/BglII & $15 \mathrm{q} 11.2-12$ & 22 & 22 & 12 & 2222 & 22 & 22 \\
\hline D15S9 & $34 / S c a I$ & $15 \mathrm{q} 11.2-12$ & 11 & 11 & 11 & 11 & 11 & 12 \\
\hline D15S11 & PCR & $15 \mathrm{q} 11.2-12$ & aa & aa & aa & $a b$ & $a b$ & $a b$ \\
\hline D15S13 & $189-1 / \operatorname{Taq} \mathrm{I}$ & $15 \mathrm{q} 11.2-12$ & 22 & 11 & 22 & - & - & - \\
\hline D15S10 & $3-21 / \operatorname{Taq} \mathrm{I}$ & $15 \mathrm{q} 11.2-12$ & 11 & 11 & 11 & - & - & - \\
\hline GABRB3 & PCR & 15 q11.2-12 & aa & bc & ad & $a b$ & $a b$ & bc \\
\hline D15S97 & PCR & 15q11.2-12 & - & - & - & $a b$ & $a b$ & bc \\
\hline GABRA5 & PCR & $15 q 11.2-12$ & aa & - & $a b$ & $a b$ & $a b$ & bc \\
\hline D15S12 & ScaI & $15 \mathrm{q} 11.2-12$ & 22 & 22 & 22 & - & - & - \\
\hline D15S24 & CMW $1 / E c o R I$ & $15 q 13$ & aa & aa & $a b$ & - & - & - \\
\hline ACTC & PCR & $15 q^{13}-15$ & aa & - & aa & - & - & - \\
\hline CYP19 & PCR & $15 \mathrm{q} 21$ & aa & ac & $a b$ & - & - & - \\
\hline D15S99 & PCR & $15 q 22-25$ & aa & - & $a b$ & - & - & - \\
\hline IPM15M9 & PCR & $15 q 25$ & aa & $\mathrm{cd}$ & $a b$ & - & - & - \\
\hline D15S86 & $\mathrm{ms} 620 / E c o R I$ & $15 q 26$ & $\mathrm{cc}$ & $\mathrm{ab}$ & bc & - & - & - \\
\hline D15S87 & PCR & $15 q 26$ & - & - & - & $a b$ & $a b$ & bc \\
\hline
\end{tabular}

$-=$ not typed.
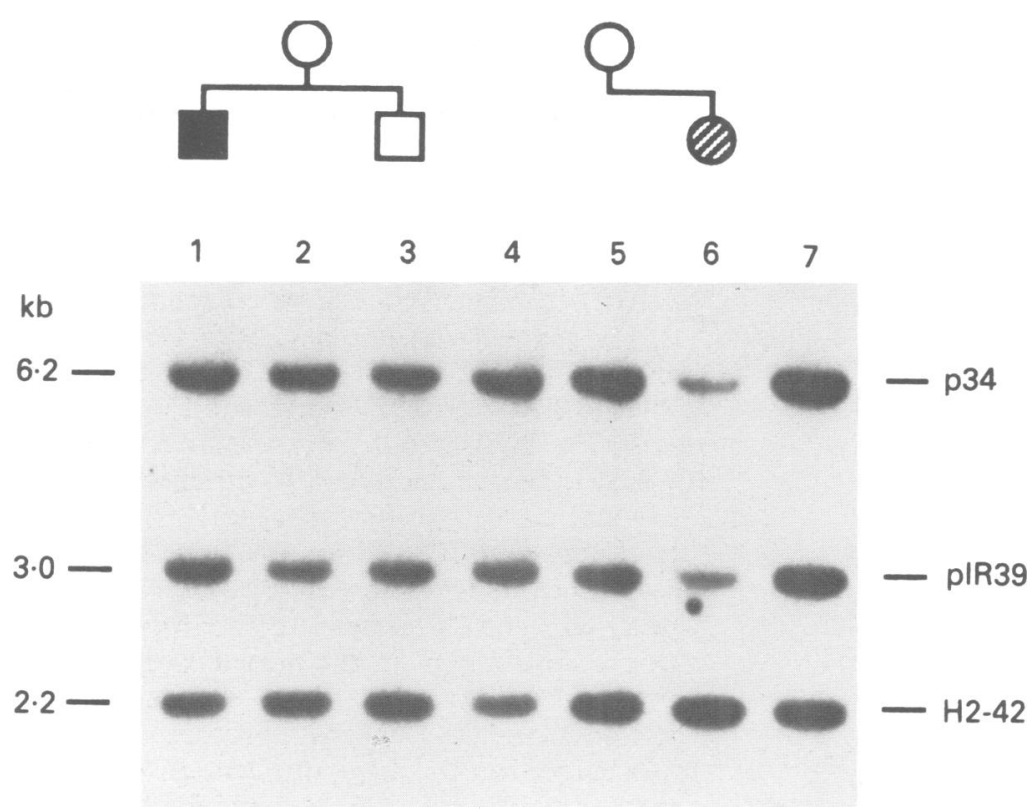

Figure 4 Dosage analysis of p34 and pIR39 in case 2 (lane 1) relative to a control, H2-42, from chromosome 13 shows a normal two copy dosage for $p 34$ but higher dosage (estimated average copy number of 3.3) for pIR39. Lane 4 is an atypical AS patient without a deletion, lane 6 is an AS deletion patient, and lane 7 is a patient with an inv dup 15 including a duplication of the $P W S / A S$ region.

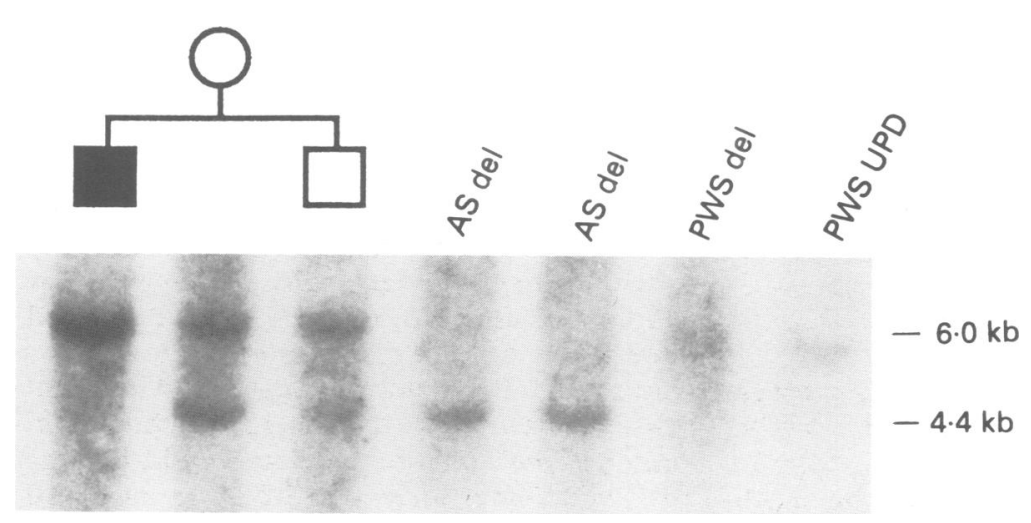

Figure 5 Parent of origin specific methylation detected by hybridisation of PW71 to HindIII/HpaII digests. Lane 1 is the PWS patient (case 2) which shows only a maternally derived methylated $6.0 \mathrm{~kb}$ band. diagnosis, exclusion of UPD(15) should be considered. UPD should also be considered as an explanation for an abnormal phenotype in patients with small marker chromosomes derived from other chromosomes. The abnormal phenotype could be the result of either duplication of genes near the centromere, or imprinting effects, or homozygosity for rare recessive mutations on the two cytogenetically normal homologues. For the majority of human chromosomes, it is still unknown what effects imprinting may have, and a search for UPD among subjects with de novo marker chromosomes seems a possible approach to determine this.

This project was supported in part by the EMDO Stiftung, Zürich (WPR and AS), Swiss National Foundation grant B 2006.295.3412 (WPR, FB, and AS), and an $\mathrm{NIH}$ clinical investigator award and a Charles $\mathrm{H}$ Hood Foundation grant to JW. We would also like to thank Marc Lalande for comments and support, Irene Sills and Robert Rapaport for endocrine evaluation of case 2, and David Ledbetter for useful discussions.

1 Knoll JHM, Nicholls RD, Magenis RE, Graham JM Jr, Lalande M, Latt SA. Angelman and Prader-Willi syndromes share a common chromosome 15 deletion but differ in parental origin of the deletion. Am $\mathcal{f}$ Med Genet 1989;32:285-90.

2 Malcolm S, Clayton-Smith J, Nichols M, et al. Uniparental disomy in the Angelman syndrome. Lancet 1991;337:6947.

3 Nicholls RD, Pai GS, Gottlieb W, Cantu ES. Paternal uniparental disomy of chromosome 15 in a child with Angelman syndrome. Ann Neurol 1992;32:512-18.

4 Mutirangura A, Greenberg F, Butler MG, et al. Multiplex PCR of three dinucleotide repeats in the Prader-Willi/ Angelman critical region (15q11-q13): molecular diagnosis and mechanism of uniparental disomy. Hum Mol geosis and mechanism

5 Nicholls RD, Knoll JHM, Butler MG, Karam S, LaLande $M$. Genetic imprinting suggested by maternal heterodisomy in non-deletion Prader-Willi syndrome. Nature 989;342:281-5.

6 Robinson WP, Bottani A, Yagang X, et al. Molecular, cytogenetic, and clinical investigations of Prader-Willi syndrome patients. Am f Hum Genet 1991;49:1219-34.

7 Mascari MJ, Gottlieb W, Rogan PK, et al. The frequency of uniparental disomy in Prader-Willi syndrome. $N$ Engl f Med 1992;326:1599-607.

8 Ridler MAC, Garrod O, Berg JM. A case of Prader-Willi syndrome in a girl with a small extra chromosome. Acta Paediatr Scand 1971;60:222.

9 Michaelsen KF, Lundsteen C, Hansen FJ. Prader-Willi syndrome and chromosomal mosaicism 46, XY/47, XY + mar in two cases. Clin Genet 1979;16:147.

10 Fujita $\mathrm{H}$, Sakamoto Y, Hamamoto Y. An extra idic(15p)(q11) chromosome in Prader-Willi syndrome. Hum Genet 1980;55:409-11. 
11 Wisniewski L, Witt ME, Ginsberg-Fellner F, Wilner J, Desnick RJ. Prader-Willi syndrome and a bisatellited derivative of chromosome 15. Clin Genet 1980;18:42-7.

12 Ledbetter DH, Mascarello JT, Riccardi VM, Harper VD, Airhart SD, Strobel RJ. Chromosome 15 abnormalities and the Prader-Willi syndrome: a follow-up report of 40 cases. Am 7 Hum Genet 1982;34:278-85.

13 Wulfsberg EA, Sparkes RS, Klisak IJ, Gurfield WB. A $(15,1)$ translocation in a patient mosaic for presence or $(15,1)$ translocation in a patient mosaic for presence or
absence of an isodic $(15 \mathrm{p})(\mathrm{q} 11)$. Am $\mathfrak{f}$ Med Genet absence of

14 Mattei JF, Mattei MG, Giraud F. Prader-Willi syndrome and chromosome 15. Hum Genet 1983;64:356-62.

15 Maraschio P, Cuoco C, Gimelli G, Zuffardi O, Tiepolo L. Origin and clinical significance of inv dup(15). In: Daniel A, ed. The cytogenetics of mammalian autosomal rearrange ments. New York: Alan R Liss, 1988:615-34.

16 Robinson WP, Binkert FB, Giné R, et al. Clinical and molecular analysis of five inv dup(15) patients. Eur f Hum Genet 1993;1:37-50.

17 Purvis-Smith SG, Saville T, Manass S, et al. Uniparental disomy 15 resulting from 'correction' of an initial trisomy 15. Am f Hum Genet 1992;50:1348-50.

18 Cassidy SB, Lai Li-Wen, Erickson RP, et al. Trisomy 15 with loss of the paternal 15 as a cause of Prader-Will syndrome due to maternal disomy. Am $\mathcal{J}$ Hum Gener 1992;51:701-8.

19 Donlon TA, Lalande M, Wyman A, Bruns G, Latt SA. Isolation of molecular probes associated with the chromoIsolation of molecular probes associated with the chromoSome 15 instability in the Prader-Wili

20 Tantravahi U, Nicholls RD, Stroh $\mathrm{H}$, et al. Quantitative calibration and use of DNA probes for investigating chromosome abnormalities in the Prader-Willi syndrome. Am $\mathcal{F}$ Med Genet 1989;33:78-89.
21 Nicholls RD, Knoll JHM, Glatt K, et al. Restriction fragment length polymorphisms within proximal $15 q$ and their use in molecular cytogenetics and the Prader-Willi syndrome. Am 7 Med Genet 1989;33:66-77.

22 Rich DC. Highly polymorphic locus D15S24 (CMW-1) maps to 15pter-q13. Nucleic Acids Res 1988;16:8740.

23 Armour JAL, Povey S, Jeremiah S, Jefferys AL. Systematic cloning of human minisatellites from ordered array chromatid libraries. Genomics 1990;8:501-12.

24 Mutirangura A, Kuwano A, Ledbetter SA, Chinault AC, Ledbetter DH. Dinucleotide repeat polymorphism at the Ledbetter DH. Dinucleotide repeat polymorphism at the PWS) of chromosome 15. Hum Mol Genet 1992;1:139.

25 Mutirangura A, Ledbetter SA, Kuwano A, Chinault AC, Ledbetter DH. Dinucleotide repeat polymorphism at the Ledbetter DH. Dinucleotide repeat polymorphism at the GABA
Prader-Willi region (AS/PWS) of chromosome 15. Hum Mol Genet 1992;1:67.

26 Glatt KA, Sinnett D, Lalande M. Dinucleotide repeat

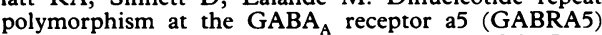
locus at chromosome 15q11-q13. Hum Mol Genet 1992;1:348.

27 Kuwano A, Mutirangura A, Dittrich B, et al. Molecular dissection of the Prader-Willi/Angelman syndrome region (15q11-13) by YAC cloning and FISH analysis. Hum Mol Genet 1992;1:417-25.

28 Robinson WP, Spiegel R, Schinzel AA. Deletion breakpoints associated with the Prader-Willi and Angelman points associated with the Prader-W illi and Angelman recombination. Hum Genet 1993;91:181-4.

29 Dittrich B, Robinson WP, Knoblauch $\mathrm{H}$, Buiting $\mathrm{K}$, Schmidt K, Gillesen-Kaesbach G, Horsthemke B. MoSchmidt K, Gillesen-Kaesbach G, Horsthemke B. Molecular diagnosis of the Prader-Willi and Angelman syn-
dromes by detection of a parent of origin specific DNA dromes by detection of a parent of origin specific DN
methylation in 15q11-13. Hum Genet 1992;90:313-15. 